\title{
Impedance-matched differential SNSPDs for practical photon counting with sub-10 ps timing jitter
}

\author{
Marco Colangelo $^{1 \#}$, Andrew Beyer ${ }^{2}$, Boris Korzh ${ }^{2 \#}$, Jason P. Allmaras ${ }^{2,3}$, Andrew Mueller ${ }^{2,3}$, Ryan M. \\ Briggs $^{2}$, Bruce Bumble ${ }^{2}$, Marcus Runyan ${ }^{2}$, Martin J. Stevens ${ }^{4}$, Adam McCaughan ${ }^{4}$, Di Zhu ${ }^{1}$, Steve Smith ${ }^{5}$, \\ Wolfgang Becker ${ }^{6}$, Lautaro Narváez ${ }^{7}$, Joshua C. Bienfang ${ }^{8}$, Simone Frasca ${ }^{2}$, Angel E. Velasco ${ }^{2}$, Edward \\ Ramirez $^{2,9}$, Alexander Walter ${ }^{2}$, Ekkehart Schmidt ${ }^{2}$, Emma E. Wollman $^{2}$, Cristián Peña ${ }^{10,7}$, Maria Spiropulu ${ }^{7}$, \\ Richard P. Mirin ${ }^{4}$, Sae Woo Nam ${ }^{4}$, Karl K. Berggren', and Matthew D. Shaw ${ }^{2}$
}

${ }^{1}$ Department of Electrical Engineering and Computer Science, Massachusetts Institute of Technology, Cambridge, MA, USA. ${ }^{2} J e t$ Propulsion Laboratory, California Institute of Technology, 4800 Oak Grove Dr., Pasadena, CA, USA. ${ }^{3}$ Applied Physics, California Institute of Technology, 1200 E California Blvd, Pasadena, CA, USA. ${ }^{4}$ National Institute of Standards and Technology, Boulder, CO, USA. ${ }^{5}$ Cosmic Microwave Technology, 15703 Condon Avenue, Lawndale, CA, USA. ${ }^{6}$ Becker \& Hickl GmbH, Nahmitzer Damm 30, Berlin, Germany. ${ }^{7}$ Division of Physics, Mathematics and Astronomy, California Institute of Technology, Pasadena, CA, USA. ${ }^{8}$ National Institute of Standards and Technology, Gaithersburg, MD, USA. ${ }^{9}$ California State University, Los Angeles, CA, USA. ${ }^{10}$ Fermi National Accelerator Laboratory, Batavia, IL, USA. ${ }^{\#}$ colang@mit.edu, bkorzh@jpl.caltech.edu

\begin{abstract}
We demonstrate large-area superconducting nanowire single-photon detectors (SNSPDs) with simultaneous high system detection efficiency and low system jitter. We describe the device architecture and discuss optimal readout setup for practical applications. C 2021 The Author(s)
\end{abstract}

Superconducting nanowire single-photon detectors (SNSPDs) are the highest performing photon-counting technology with demonstrations of $98 \%$ efficiency at telecom wavelengths [1], ultra-low intrinsic dark count rates [2,3], single element count rates $>100 \mathrm{MHz}$ [4], intrinsic timing jitter as low as $2.6 \mathrm{ps}$ to $4.3 \mathrm{ps}$ [5] depending on the wavelength, as well as intrinsic photon number resolution [6].

Combining several of these metrics into a single device can impact several fields such as quantum communication, laser ranging, low-power optical waveform capture and deep space optical communication, yet it remains a significant challenge. One of the main difficulties is the combination of a large active area with low jitter operation, due to geometric contributions to the timing of the readout signal. Additionally, introduction of low-jitter SNSPDs into applications requires high performance time-tagging and signal conditioning. Most investigations typically make use of high-bandwidth oscilloscopes to characterize only the detector jitter, but a demonstration of low system jitter for practical measurement is desired.

In this paper we show that by engineering the detector architecture and the readout acquisition setup we achieve high-performance in several detector metrics at the same time. We demonstrate large-area impedance-matched differential detectors with less than 10 ps system jitter and $>45 \%$ system detection efficiency. Our detectors are also intrinsically capable of single-pixel imaging and photon number resolution up to three photons.

Our SNSPD consist of a conventional thin-film niobium nitride $(\mathrm{NbN})$ nanowire meander covering an area larger than $10 \mu \mathrm{m} \times 15 \mu \mathrm{m}$, embedded in a dielectric cavity (tuned to the target detection wavelength) with one dielectric mirror and one metal mirror (Fig. 1a-d). Due to the high kinetic-inductance of thin-film NbN and the local ground plane introduced by the cavity metal reflector, the nanowire acts as a high-impedance low-speed transmission line. These distributed effects generate two main issues. (1) The location-dependent delays induced by the slow phase velocity create an additional timing uncertainty on the pulse arrival time (longitudinal geometric jitter $j_{\text {geom }}$ ). (2) The impedance mismatch between the nanowire and the $50 \Omega$ low-noise amplifier induces multiple reflections and distortion of the output pulses. The signal-to-noise ratio (SNR) is degraded, and the electrical noise contribution to the timing jitter increases. Our proposed detector and readout designs are aimed at mitigating the above issues.

We monolithically integrate two Klopfenstein impedance matching tapers, one for each end, to interface the highimpedance nanowire to the $50 \Omega$ readout electronics (Fig. $1 \mathrm{~b}$ and $1 \mathrm{c}$ ). The impedance taper minimizes reflections, increases the pulse amplitude and the slew-rate, and allows a reduction of the electrical noise contribution to the timing jitter through a higher SNR.

To compensate for the geometric effects induced by the slow phase velocity, the detector is designed in a differential configuration (Fig. 1e). A detection event generates two complementary pulses which can be individually acquired through a real-time high-bandwidth oscilloscope. The single-ended time-tags $t_{1}$ and $t_{2}$ are strictly dependent on the detection coordinate, but their sum $t_{\Sigma}=\left(t_{1}+t_{2}\right) / 2$ depends only on the detection time. The jitter of the sum time $j_{\Sigma}$ compensates the geometric contribution and represents the true timing resolution [7].

In many photon-counting applications it is not practical to use two, low jitter, time taggers per detector. To overcome this, while exploiting the benefits of geometric jitter cancellation in a differential configuration, we designed a readout scheme that makes the differential detector compatible with a single-ended, time-correlated single photon counting 
(TCSPC) module. As shown in Fig. 1f, when the complementary pulses' waveforms are available, $t_{\Sigma}$ can be equivalently obtained as the time-tag of the difference of the complementary waveforms, which, to avoid confusion, we call $t_{\text {diff. }}$.

In practice, as shown in Fig. 1g, the complementary pulses are first fed to a $3 \mathrm{GHz} 2: 1$ balun transformer, which performs a complementary hardware subtraction. The output of the balun is then connected to a TCSPC card which extracts the time-tag $t_{\text {diff. }}$ The jitter on $t_{\text {diff }}$ still has the longitudinal geometric contribution compensated for and represents the timing resolution of the entire measurement system.

Fig. 2 shows the characterization results for a representative detector when illuminated with an attenuated $775 \mathrm{~nm}$ light source with $\approx 1$ ps pulse width and $10 \mathrm{MHz}$ repetition rate. The active area is $10 \mu \mathrm{m} \times 15 \mu \mathrm{m}$ and the cavity is tuned for detection at $775 \mathrm{~nm}$. The system detection efficiency is $47 \%$ (Fig. 2a). At $22 \mu \mathrm{A}$ ( $98.9 \%$ of the switching current) the detector jitter $j_{\Sigma}$ is 9.4 ps. The detection efficiency for this particular device is limited by slight misalignment of the optical fiber. When the fiber is aligned the system detection efficiency should exceed $70 \%$. The system jitter characterized with the setup consisting of the balun and the single-ended TCSPC module is $9.7 \mathrm{ps}$. The slight $0.3 \mathrm{ps}$ degradation is attributed to the insertion loss of the balun $(6 \mathrm{~dB})$ which increases the impact of the electrical noise on the timing resolution.

Our differential impedance-matched detector, coupled with the differential-to-single-ended readout combines high system detection efficiency with low system jitter while keeping the convenience of a single-ended readout. Our detectors provide a practical solution for high-performance single photon counting with potential impact on fields such as quantum information science, deep-space optical communication and biology.
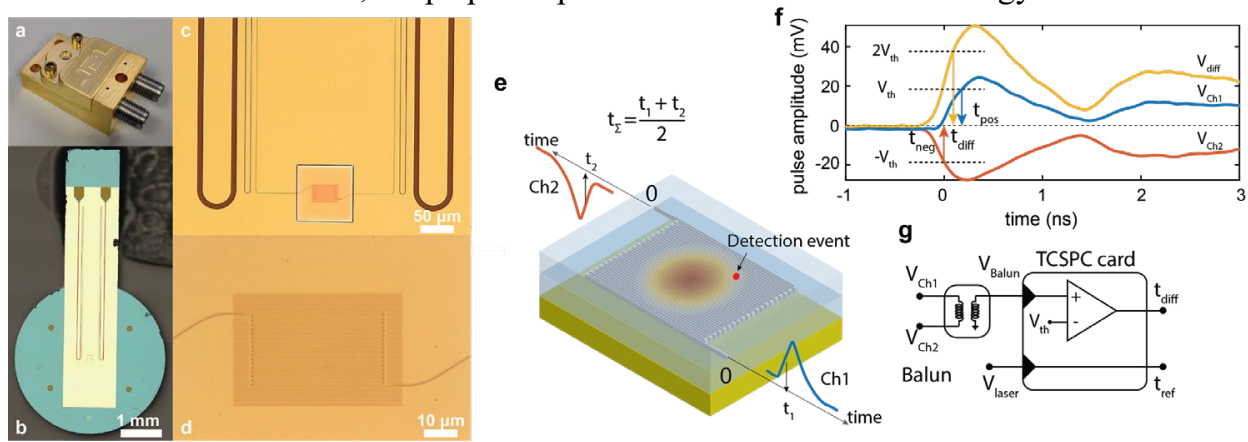

Figure 1: (a) Picture of the packaged detectors. (b) Optical micrograph of the detector die: the lollipop-shaped die is obtained through deep reactive ion etching and it is used for the self-aligned fiber-coupled packaging. (c) Optical micrograph of the nanowire meander embedded in the optical cavity and differential tapers. (d) Optical micrograph of the active area. (e) Working principle of a differential detector. A detection event generates two complementary pulses which can be acquired and processed to obtain two time-tags, whose sum, $t_{\Sigma}$, allows cancellation of the geometric jitter. (f) The difference of the complementary pulses can be processed to obtain a time-tag, $t_{\mathrm{diff}}$, where $t_{\mathrm{diff}} \approx t_{\Sigma}$. $(\mathrm{g})$ Hardware implementation of the processing in (e). The complementary pulses are fed to a 2:1 balun transformer. The balun output is fed to a single-ended

TCSPC card. The differential geometric jitter cancellation is achieved while keeping the convenience of a single-ended readout setup.

a

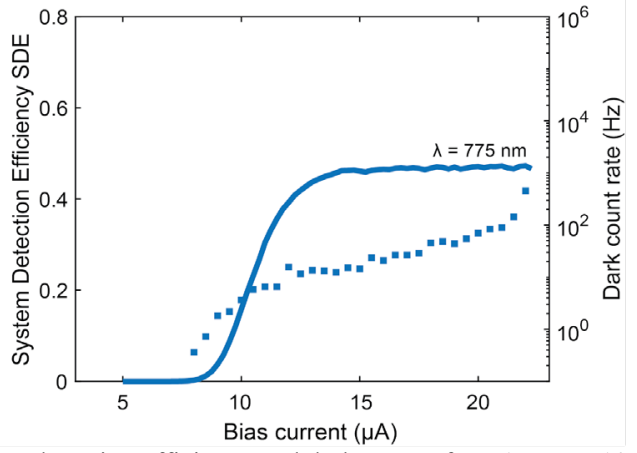

b

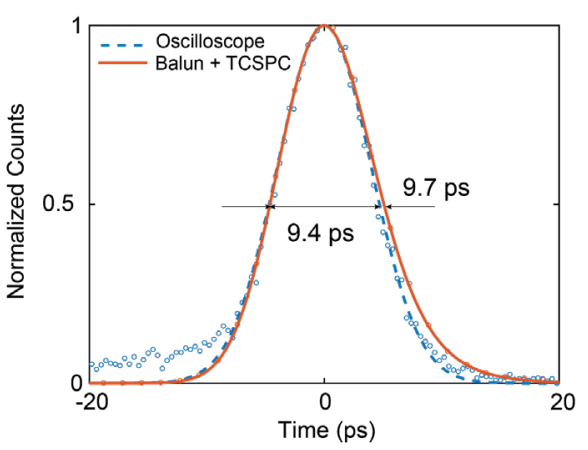

Fig.2: (a) System detection efficiency and dark counts for a $15 \mu \mathrm{m} \times 10 \mu \mathrm{m}$ differential matched detector optimized for $775 \mathrm{~nm}$ photons. (b) Comparison of the detector sum jitter $j_{\Sigma}$, obtained with a high-resolution real-time oscilloscope, and the system jitter $j_{\text {diff }}$, obtained through a 2:1

\section{References} balun combined with a TCSPC card.

[1] D.V. Reddy et al. Optica 7.12 (2020): 1649-1653. [2] E. E. Wollman et al. Optics express 25.22 (2017): 26792-26801. [3] Y. Hochberg et al. Physical review letters 123.15 (2019): 151802. [4] A. J. Kerman et al. Journal of Applied Physics 113.14 (2013): 144511. [5] B. Korzh et al. Nature Photonics 14.4 (2020): 250-255. [6] D. Zhu et al. Nano Letters 20.5 (2020): 3858-3863. [7] N. Calandri et al. Applied Physics Letters 109.15 (2016): 152601. 